\title{
Participação política e gestão fiscal explicam desenvolvimento social nos municípios brasileiros?
}

\author{
Welles Matias de Abreu \\ Universidade de Brasília (UnB)
}

Ricardo Corrêa Gomes

Universidade de Brasília (UnB)

Daniel Cerqueira Ribeiro

Universidade de Brasília (UnB)

José Matias-Pereira

Universidade de Brasília (UnB)

Fábio Jacinto Barreto de Souza

Universidade de Brasília (UnB)

Este artigo tem como objetivo central verificar se variáveis relacionadas com participação política e gestão fiscal explicam significativamente o desenvolvimento social nos municípios. Para tanto, observa-se que o processo participativo fortalece a promoção de iniciativas inclusivas, enquanto a gestão fiscal garante o cumprimento em especial da função estabilizadora, com vistas a promover o desenvolvimento social. Sob a perspectiva metodológica, trata-se de uma pesquisa ex-post-facto, a qual tem o propósito de analisar quantitativamente se há relação de significância entre as variáveis da pesquisa (participação política, gestão fiscal e desenvolvimento social). O resultado principal da pesquisa foi a constatação de significância estatística validando a existência de relação positiva do desenvolvimento social com a participação política e gestão fiscal. Após a adição de variável controle relacionada com a questão regional, pôde-se identificar a ocorrência de ganhos significativos de explicação do modelo em estudo. Assim sendo, instituições participativas politicamente e responsáveis do ponto de vista fiscal, além de melhorar a qualidade democrática, apresentam um círculo virtuoso buscando a redução da pobreza, rumo ao desenvolvimento social.

Palavras chave: participação política, gestão fiscal, desenvolvimento social

[Artigo recebido em 9 de junho de 2016. Aprovado em 29 de agosto de 2017.] 


\section{¿Participación política y gestión fiscal explican el desarrollo social en los municipios brasileños?}

Este artículo está dirigido principalmente para verificar que las variables relacionadas con la política y la participación de la gestión fiscal explican en gran medida el desarrollo social en los municipios. Por lo tanto, se observa que el proceso de participación fortalece la promoción de iniciativas incluyentes, mientras que la gestión fiscal asegura el cumplimiento, en particular, la función de estabilización, con el fin de promover el desarrollo social. Bajo el punto de vista metodológico, se trata de una investigación ex post facto, que está destinada a analizar cuantitativamente si existe una relación significativa entre las variables de la investigación (participación política, la gestión fiscal y el desarrollo social). El resultado principal del estudio fue el hallazgo de significación estadística validar la existencia de una relación positiva entre el desarrollo social con la participación política y la gestión fiscal. Se observó que después de la adición de control variable relacionada con la pregunta regional, puede identificar la aparición de aumentos significativos en el modelo bajo explicación estudio. Por lo tanto, las instituciones políticas de participación y el punto de vista fiscal responsable, además de mejorar la calidad de la democracia, tienen un círculo virtuoso que busca la reducción de la pobreza, al desarrollo social.

Palabras clave: desarrollo social, administración municipal, participación social, gestión fiscal, gestión participativa

\section{Do political participation and fiscal management explainsocial development in Brazilian municipalities?}

The article's aim is to verify if political participation and fiscal management explain social development of Brazilian municipalities. The participatory process strengthens the promotion of inclusive initiatives, while fiscal management ensures the stabilizer function, in order to promote social development. Under the methodological perspective, this is an ex-post-facto research, which is intended to verify, quantitatively, the existence of significant relationship between the variables: political participation; fiscal management; and social development. The main result of this study was the finding of statistical significance that exist a positive relationship of social development with political participation and fiscal management. In addition, it was observed that adding a control variable related to the regional issue, we should identify the occurrence of explanation gains. In a nutshell, participatory political institutions and responsible fiscal perspectives can promote a virtuous circle focus on reduction of poverty, in order to improve social development.

Keywords: social development, municipal administration, social participation, fiscal management, participatory management 


\section{Introdução}

Instituições participativas visam melhorar a governança, o empoderamento dos cidadãos, bem como a qualidade da democracia, criando um ciclo virtuoso para reduzir a pobreza e promover o desenvolvimento social (TOUCHTON; WAMPLER, 2014). Algumas correntes teóricas defendem a participação política democrática e argumentam que essa participação busca superar as desigualdades materiais, os déficits de opinião e de vontade política, e expandir o processo democrático da esfera política para a esfera econômica, tendo assim, também, um caráter redistributivo (PATEMAN, 1992; COELHO; NOBRE, 2004; RODRIGUEZ et al., 2004; MARQUETTI et al., 2008). No entanto, é importante ressaltar que os recursos públicos são essenciais para a implantação das políticas públicas prioritárias, seja em processos participativos ou não. Dessa forma, a gestão fiscal deve também ter a sua importância por si só para a promoção do desenvolvimento social (ALBUQUERQUE et al., 2006; FrIIS-HANSEN; KYED, 2009; GRUBER, 2009). Nesse contexto, o presente artigo busca verificar se a participação política e a gestão fiscal estão relacionadas com o desenvolvimento social municipal.

Para tanto, o objetivo da pesquisa é verificar se variáveis relacionadas com participação política e com gestão fiscal explicam o desenvolvimento social nos municípios. Assim sendo, espera-se checar se a interação entre o interesse da sociedade local em participar do processo político democrático e a capacidade do governo em gerir adequadamente as questões fiscais municipais promovem desenvolvimento social.

É importante ressaltar que as condições referentes aos interesses, poder e urgência dos stakeholders devem estar associadas a um processo democrático de governança (FreEMAN, 1984; 1994; Mitchell et al., 1997; ANSELL; GASH, 2008), com capacidade para solucionar problemas públicos, que demandam recursos fiscais, como os relacionados com: saúde, segurança, educação, habitação, além dos associados com as desigualdades social e regional (Provan; MILWARD, 1995; BovaIRD, 2005; Provan; KenIS, 2008; WORLD BANK, 2014). Dessa forma, a pesquisa em questão é fundamental, uma vez que é premente checar a relação entre o desenvolvimento social com a participação social e a gestão fiscal.

O presente trabalho está dividido em quatro partes. Na primeira consta o referencial teórico sobre participação política, gestão fiscal e desenvolvimento social. Na segunda, são discriminados os aspectos metodológicos da pesquisa. $\mathrm{Na}$ terceira, são expostos os resultados e discussões. E na quarta, e última parte, são apresentadas as considerações finais sobre o estudo em comento. 


\section{Referencial teórico}

O referencial teórico deste trabalho utiliza as contribuições relacionadas com a participação política, gestão fiscal e desenvolvimento social. O processo participativo fortalece a promoção de iniciativas inclusivas (LIJPHART, 2003), enquanto a gestão fiscal garante o cumprimento em especial da função estabilizadora (ALBUQUERQUE et al., 2006), com vistas a promover o desenvolvimento social (SHAH, 2007; BRASIL, 2013).

Há duas condições fundamentais para garantir os direitos constitucionais dos brasileiros, atingindo o requerido nível de desenvolvimento social: a primeira se refere ao necessário crescimento econômico, ou seja, está relacionada com o elemento gestão fiscal; e o segunda é o engajamento político da sociedade, de forma a diminuir os custos da participação eleitoral e, consequentemente, direcionar as decisões governamentais visando a políticas públicas inclusivas, como forma de buscar a redução das desigualdades sociais (SANTOS et al., 2005). Dessa forma, apresenta-se, a seguir, maiores detalhes teóricos, bem como as suas respectivas interações e suas principais contribuições para o desenvolvimento da pesquisa.

\section{Participação política}

Participação é definida como o empoderamento político e um incentivo para o engajamento dos stakeholders, assim como se refere à emancipação da autoridade de tomada de decisões e do acompanhamento aos agentes não governamentais (SHAH, 2007; KHAGRAM et al., 2013; LING; RoBERTS, 2014). A participação ajuda a fortalecer a sociedade civil por meio de mecanismos institucionais no âmbito dos processos decisórios de políticas públicas indiretas e diretas, por exemplo, por meio de delegação a líderes no âmbito do Poder Legislativo ou em conselhos representativos, bem como através da participação dos próprios cidadãos (BAIOCCHI et al., 2006; SHAH, 2007; FrIIS-HANSEN; KYED, 2009; KASYMOVA; SCHACHTER, 2014; LING; ROBERTS, 2014). O processo participativo fortalece a indução de iniciativas inclusivas, fornecendo aos cidadãos marginalizados e excluídos o direito de ter suas vozes ouvidas e colaborar com influências nas decisões governamentais (YILMAZ et al., 2010; WAMPler, 2012; GaVenta; McGee, 2013; KASYMOVA; SchaCHTER, 2014; LING; ROBERTS, 2014).

Dessa forma, as regras e as estruturas de representação política local criam incentivos (positivos ou negativos) de articulação e agregação de interesses dos cidadãos, como forma de estímulo para o engajamento de líderes, com vistas a servirem de "juízes" promotores de consensos no âmbito dos conflitos existentes no momento da tomada de decisão (SHAH, 2007; LING; ROBERTS, 2014). No entanto, é importante destacar que o debate sobre quem deve representar a sociedade civil deve 
preceder a adoção de instituições participativas; nesse sentido, é com a incorporação do sentimento da importância de participar de uma decisão que se deve esperar que a sociedade civil possa engajar efetivamente nas ações governamentais, aumentando, assim, suas vozes sobre a política pública (WAMPLER, 2012).

A participação política pode ser observada de diversas maneiras, e é considerada como uma das medidas mais importantes no âmbito de uma democracia (ALTMAN; Pérez-LIÑÁN, 2002; DAHL, 2012). Nenhum regime pode ser considerado democrático se não garantir para todos os cidadãos adultos direitos formais de participação política, incluindo o direito ao voto, bem como igualdade legislativa (LAWRENCE, 1997; NYE et al., 1997; THE ECONOMIST, 2014).

De acordo com Diamond e Morlino (2004), a participação dos cidadãos não está ligada somente ao voto, mas se dá através do envolvimento no processo político, organizando-se em grupos, comunicando suas demandas, responsabilizando ou "premiando" os seus representantes e envolvendo-se em questões públicas. Porém, nem todos estão dispostos a se envolver. As instituições participativas precisam de cidadãos ativos, sendo que nas democracias mais consolidadas, esse envolvimento de cidadãos pode ser presenciado em mais da metade dos que têm direito ao voto (Воввıо, 1986; Воввіо; Bovero, 1986). Assim, a participação pode melhorar a alocação de recursos orçamentários, reforçando e gerando melhorias democráticas e de ordem social, em especial aquelas voltadas à redução da pobreza (CABANNES, 2004; WAMPLER, 2004; NAVARRO, 2005; SHAH, 2007; WAMPLER, 2012).

Em uma democracia plena, todos os cidadãos devem ter direitos iguais de participação nas decisões políticas. Todas as pessoas que são afetadas por uma decisão política devem ter o direito de participar na formação daquela decisão em iguais condições (Bühlmann et al., 2012). Assim, não deve haver lacunas de participação no que se refere a recursos de poder ou características sociais. Existem diversas formas de se analisar a participação. De acordo com Lijphart (2003), a participação é um excelente indicador de democracia, pois mostra o grau de interesse dos cidadãos para serem representados. Para ele, esse indicador está fortemente relacionado ao status socioeconômico e, por esse fato, também pode servir de indicador indireto de igualdade política.

Os parâmetros de análise sugeridos por Lijphart (2003) para participação são o número de eleitores como percentagem da população votante, a percentagem de participação em eleições que tenha alcançado o mais alto índice de comparecimento. Segundo o autor, a partir da análise de dados específicos, a Itália teve o mais alto nível de comparecimento, enquanto a Suíça teve o mais baixo, 40,9\%, no período de 1971 a 1996. A obrigatoriedade do voto, o nível de desenvolvimento do país e 
a frequência de escolhas eleitorais a serem feitas afetam significativamente esse indicador (LIJPHART, 2003)

Em alguns casos, esse indicador pode refletir o direito de participar, mas não a taxa real de participação (SANTOS et al., 2005). Alguns estudos, sobretudo os trabalhos ligados à corrente da democracia deliberativa, argumentam que o número de eleitores não é o melhor parâmetro para se definir uma democracia, pois o mais importante, para esses autores, seria o processo comunicativo entre diversos atores políticos, em que, a partir do poder argumentativo, chegar-se-ia a um consenso. A partir do momento em que esses atores participassem dessas arenas de debate, possuíssem poder de voz, não só para expor suas demandas, mas também para argumentar, o processo democrático passaria a ser mais efetivo (AVRITZER; NAVARRo, 2003; HABERMAS, 2012). Todavia, há significativa literatura que destaca o comparecimento eleitoral como um parâmetro importante da associação ao processo democrático, uma vez que permite captar a vontade de participação política, seja ela voluntária ou incentivada pelo voto obrigatório (AltMAN; PÉrEZ-LIÑÁN, 2002).

Assim sendo, a participação precisa captar o interesse dos cidadãos em estar presente na definição, por exemplo, de suas representações democráticas, que devem promover os consensos com vistas a focar os interesses coletivos, de forma colaborativa e inclusiva, buscando a justiça social (SCHNEIDER; BAQUERO, 2006; SCHROEDER, 2010). Por fim, espera-se que quanto maior for a participação política, melhores serão os resultados de desenvolvimento social (CABANNES, 2004; WAMPLER, 2004; NAVARRO, 2005; SHAH, 2007), considerando inclusive a expressiva relação com a redução da pobreza extrema e da mortalidade infantil (BAIOcCHI et al., 2006).

\section{Gestão fiscal}

As instituições orçamentárias têm um papel relevante na modelagem do desempenho econômico, uma vez que participam da definição e aplicação de regras da economia de um país (NORTH, 1990; HYDE, 2002). Cabe registrar que as instituições, como regras do jogo, são concebidas pelo homem, a partir da sua própria estrutura de interação, quer sejam formais (por exemplo, as leis) ou informais (como normas de comportamento, códigos e convenções de conduta), bem como apresentam características relacionadas com a sua aplicação, observadas as crenças compartilhadas sobre o mundo (NORTH, 1991).

Sobre o processo orçamentário, a elaboração mais comum e melhor constituída das teorias da escolha racional é a que considera a incerteza ao redor das 
consequências futuras às ações presentes. Isso porque tal elaboração parte do pressuposto de que as decisões orçamentárias ocorrem sob uma limitada possibilidade cognitiva para o levantamento de alternativas (PADGET, 1980).

Em um processo decisório de alocação de recursos no orçamento federal, as decisões correspondem à própria formulação de estratégia para o desenvolvimento, bem como dependem do cálculo de quais alternativas considerar para escolher. Cabe ressaltar a complexidade do cálculo que abarca as decisões orçamentárias, considerando a grande quantidade de itens envolvidos, tendo em vista haver pouco ou quase nenhum suporte em teorias que possam antever as consequências das alternativas (fundamentadas em preferências) ou a probabilidade de sua ocorrência (WILDAVSKY; DEMPSTER; DAVIS, 1966).

As decisões orçamentárias em geral atendem a uma perspectiva incrementalista, em que se pressupõe que a solicitação da dotação orçamentária de um ano é composta por uma parcela fixa e outra variável. A fixa é considerada o alicerce orçamentário, significando o resultado de um acordo político no qual se estabelece o nível de participação de cada ação no conjunto geral (BARCELOS, 2008). Portanto, as decisões orçamentárias ocorrem, na maioria das vezes, de forma incremental. Isso faz com que o processo orçamentário se torne menos oneroso e mais estável e previsível (SWAIN; HARTLEY JR, 2001).

Especificamente sobre o processo orçamentário brasileiro, destaca-se que ele sofreu forte influência internacional, com vistas a conferir aos governos uma gestão pública enxuta, mais eficiente e efetiva, como forma de garantir ínfima governabilidade e proceder a uma extensa reforma do aparelho do Estado para o modelo gerencial, a partir do burocrático (CORE, 2004). Nesse sentido, a reforma orçamentária brasileira de 2000 teve como pontos-chave, para a sua implantação, mudanças com finalidades gerenciais no processo orçamentário (GARCIA, 2000), quer seja no processo de elaboração do Plano Plurianual (PPA) e da Lei Orçamentária Anual (LOA), ou na melhoria da gestão fiscal no âmbito da Lei de Diretrizes Orçamentárias (LDO).

A respeito da gestão fiscal brasileira, considera-se que o conjunto de ações fiscais é aquele em que a administração pública toma as decisões sobre as despesas e as receitas com vistas a garantir o atendimento da função estabilizadora, cabendo destacar que o uso equilibrado dos recursos públicos (com constantes superávits fiscais) visa, principalmente, à redução da dívida com relação ao percentual do produto interno bruto (PIB), de maneira gradual (AlbuQUERQUE et al., 2006; GRUBER, 2009; ABREU, 2013). Embora a gestão fiscal encontre-se bem orientada na estabilidade econômica, não se pode deixar de lado a preocupação com as funções orçamentárias relacionadas com a distribuição de renda e a alocação de recursos 
(GRUBER, 2009).

Agestão fiscal responsável (que contempla a obtenção de metas fiscais constantes para superávits orçamentários primários) tem como objetivo condicionar a ação estatal aos rigorosos limites de sua efetiva capacidade de gasto governamental, de forma a evitar déficits fiscais e, por seguinte, o descontrole das contas públicas (Albuquerque et al., 2006; Gruber, 2009).

No Brasil, independentemente do ente governamental, o uso equilibrado dos recursos públicos visa, além de à redução gradual da dívida como percentual do PIB (de forma a contribuir com a estabilidade), a também possibilitar o crescimento econômico com desenvolvimento social do país, com a criação de empregos, o incremento dos investimentos públicos e o aumento da rede de seguridade social, com foco na redução da desigualdade e da pobreza (AlBuQUERQUE et al., 2006; ABREU, 2013; BRASIL, 2013).

Segundo Cara (2012) e Cruz et al. (2012), pode-se utilizar o índice Firjan de gestão fiscal (IFGF), da Federação de Indústrias do Estado do Rio de Janeiro (Firjan), com vistas a obter dados sobre a gestão fiscal municipal. O IFGF visa contribuir com uma gestão pública eficiente e democrática dos municípios, e apresenta como subcomponentes dados relacionados com: receita própria, gastos com pessoal, investimentos, liquidez e custo da dívida (FEDERAÇÃo DE INDÚSTRIAS DO ESTADO DO RIO DE JANEIRO, 2014).

\section{Desenvolvimento social}

De acordo com Davis (2004), desenvolvimento social significa melhorar o bemestar e da qualidade de vida dos indivíduos por meio da introdução de mudanças institucionais visando buscar uma transformação social, possibilitando que se obtenha uma sociedade inclusiva para todos e mais justa, em um modelo de governança pública. Nesse sentido, o desenvolvimento social necessita priorizar as pessoas nos processos de desenvolvimento, dando-lhes voz e priorizando a promoção de políticas públicas sociais participativas (WORLD BANK, 2014).

Segundo o Department for International Development (DFID) (2006, p. 2), a governança é "sobre poder, autoridade e como um país gere os seus assuntos". Então, devemos esperar que a boa governança esteja relacionada com a capacidade de prevenção de conflitos violentos, o cumprimento das obrigações de direitos humanos, a melhoria da prestação de serviços públicos essenciais aos seus cidadãos. Dessa forma, a governança deve melhorar a capacidade da gestão fiscal governamental, assim como aumentar o poder de voz dos cidadãos (ABBAD; TORRES, 2002).

Registra-se que a governança está associada ao processo decisório baseado 
em instituições, considerando as vozes dos cidadãos e preocupações públicas estratégicas, diante das restrições de recursos impostas (STOKER, 1998; BOVAIRD, 2005). A governança colaborativa preconiza que processos e estruturas da tomada de decisão devem buscar envolver a sociedade para participar de forma construtiva, buscando a melhoria das condições sociais de toda a população (ANSELL; GASH, 2008; EMERSON; MURCHIE, 2011; EMERSON et al., 2012).

Nesse sentido, iniciativas para aumentar a participação política (dando voz aos cidadãos e promovendo o engajamento), bem como ações governamentais associadas com a gestão fiscal (garantindo o incremento da capacidade de financiamento das políticas públicas), estão associadas com a geração de impactos positivos no desenvolvimento social (Albuquerque et al., 2006; DEPARTMENT FOR INTERnAtional DEVELOPMENT, 2006; SHAH, 2007; FRIIS-HANSEN; KYED, 2009; GRUBER, 2009; BRASIL, 2013; FRIIS-HANSEN; Cold-RAVNKILDE, 2013; LING; RoBERTS, 2014).

De acordo com Postali e Nishijima (2011), Nunes et al. (2013), Silva (2013) e Cara (2012), o desenvolvimento social dos municípios brasileiros é aferido pelo índice Firjan de desenvolvimento municipal (IFDM), da Firjan. O IFDM tem como subcomponentes dados sobre a educação, a saúde e a renda dos municípios (FEDERAÇÃO dE INDÚSTRIAS do ESTADO do RIO dE JANEIRO, 2014). Complementarmente, registra-se que a divisão regional é basilar para subsidiar a formulação de políticas públicas, quais sejam, as associadas com as decisões para a implementação de atividades econômicas, as sociais e as tributárias, bem como de planejamento e estudos, baseados no desenvolvimento social, visando reduzir a pobreza e, consequentemente, as desigualdades entre as regiões (BRASIL, 2014).

Dessa forma, as políticas públicas que visam ao desenvolvimento social local no Brasil são formuladas tendo como base as divisões regionais (sendo a base os municípios e o teto as regiões) para as definições políticas (BRASIL, 2013, 2014). Portanto, as estratégias participativas e orçamentárias são focadas na formação de instituições públicas visando promover o desenvolvimento social municipal, no entanto deve-se levar em consideração, também, as suas respectivas dimensões regionais como base de controle das ações governamentais (BANDEIRA, 1999).

\section{Interações e contribuições teóricas}

Conforme apresentado no referencial teórico, espera-se que tanto a participação política quanto a gestão fiscal contribuam para o desenvolvimento social. Nesse sentido, visando à melhor visualização das interações entre as referidas teorias, apresenta-se, em seguida, o modelo teórico (Figura 1) que visa dar suporte para o atingimento do objetivo deste artigo. Adicionalmente, são relacionadas as principais 
contribuições teóricas identificadas que suportam a execução do presente trabalho.

Figura 1 - Modelo teórico

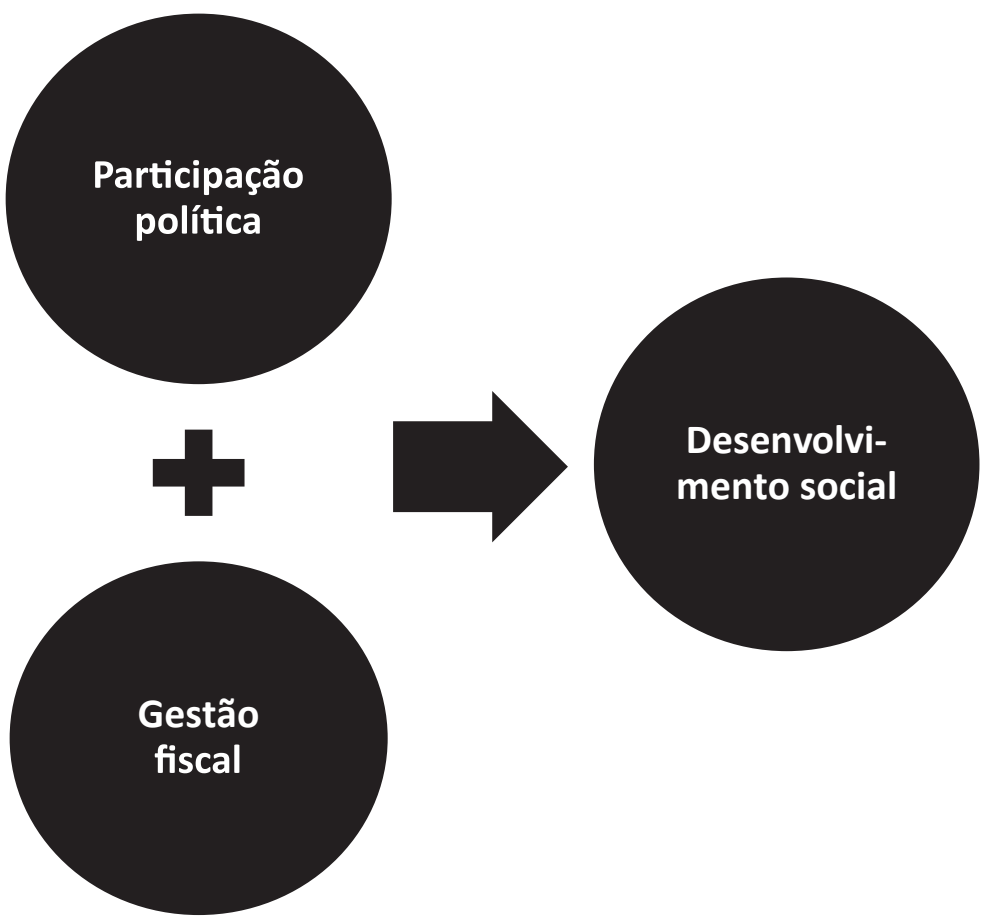

Fonte: elaboração própria.

Enquanto a participação política precisa captar o interesse dos cidadãos em estar presente na definição da representação democrática que deve conduzir a formação dos consensos sob a ótica dos interesses coletivos, a gestão fiscal visa condicionar a ação estatal de forma responsável dentro dos limites estritos da sua efetiva capacidade governamental de gasto.

Já o desenvolvimento social diz respeito à melhoria do bem-estar e da qualidade de vida dos indivíduos, por meio da promoção de mudanças institucionais, buscando a transformação social. Dessa forma, espera-se que o presente referencial teórico colabore com a busca da resposta à questão de pesquisa proposta neste trabalho.

Nesse sentido, as estratégias que estimulam a participação política e a gestão fiscal devem ser direcionadas para a promoção de mudanças institucionais que visem ao desenvolvimento social local. Sugere-se, assim, que as respectivas condições institucionais presentes em cada região brasileira devem ser levadas em consideração. 


\section{Procedimentos metodológicos}

Este artigo examina se a participação política e a gestão fiscal estão associadas com o desenvolvimento social dos municípios brasileiros. Para tanto, utilizase de uma metodologia ex-post-facto com o propósito de analisar as variáveis apresentadas a seguir, observado o modelo teórico em comento, e em conformidade com os seus respectivos índices (todos com amplitudes de 0 a 1 , com resultados positivos crescentes).

Para operacionalizar a pesquisa, utilizou-se uma variável dependente e duas variáveis independentes. Como variável dependente, o desenvolvimento social foi medido por meio do índice Firjan de desenvolvimento municipal (IFDM), cujos dados mais recentes disponíveis são de 2011. O IFDM tem componentes relacionados com a educação, a saúde e a renda dos municípios, e está disponível no sítio eletrônico da Firjan. As variáveis independentes são: a) participação política (PART) - apurada por meio da divisão do número de eleitores que compareceram pelo total de eleitores aptos para votarem na última eleição municipal brasileira (PART), no ano de 2012, obtidos no sítio do Tribunal Superior Eleitoral; e b) gestão fiscal - com base no índice de gestão fiscal (IFGF) gerado pela Firjan, com dados mais recentes de 2011, sendo os seus componentes a receita própria, os gastos com pessoal, os investimentos, bem como a liquidez e o custo da dívida.

Para a identificação da consistência regional, considerando a Região Norte (NO) como referência para análise, utilizam-se variáveis dummies, respectivamente, para controlar valores relacionados a cada uma das seguintes regiões: Nordeste (NE); Centro Oeste (CO); Sul (SU); e Sudeste (SD). Dessa forma, propõe-se a realização de duas regressões múltiplas lineares, sendo a reduzida apenas com as variações de desenvolvimento social, participação e gestão fiscal, e a completa com a inclusão das supracitadas variáveis dummies (NE, CO, SU e SD) para a análise comparativa das suas respectivas variações.

Mesmo que as mencionadas observações dos índices utilizados não estejam disponíveis no mesmo ano, as ocorrências estão bastante próximas, além de que são os dados mais recentes disponíveis. Este estudo se aplica na área de administração pública, procurando identificar a existência de relação entre o IFDM e os PART e IFGF, a partir de 5.119 ocorrências de municípios, já excluídos os casos pairwise. Espera-se que a introdução das variáveis dummies resulte em um maior poder de explicação, uma vez que a questão regional (controlada pelas variáveis dummies em comento) é utilizada como base para a elaboração das políticas públicas de desenvolvimento brasileiro. 
Dessa forma, podem-se analisar as relações do desenvolvimento social com a participação política e gestão fiscal, inclusive sobre as perspectivas regionais, utilizando os aspectos referentes à utilização da técnica estatística de regressão múltipla linear, com o apoio do aplicativo computacional STATA 13. Em se tratando de estudo com características inovadoras, vale a pena ressaltar a sua importância não apenas por apresentar resultados inéditos, mas também por servir como instrumento encorajador (estimulador) para que outras pesquisas sejam desenvolvidas sobre o tema em questão, com vistas a aperfeiçoar ou contrapor os resultados obtidos neste artigo.

\section{Resultados e discussões}

Apresentam-se, na Tabela 1, os resultados das duas regressões descritas na metodologia deste estudo, a primeira chamada de reduzida, com as variáveis IFDM, PART e IFGF, e a segunda, denominada completa, com a adição das variáveis dummies para controlar as variações por regiões. Registra-se que, tendo em vista a presença do problema de heterocedasticidade nos resíduos de ambas as regressões, optou-se pela execução das regressões utilizando a técnica de matriz robusta para tratamento do citado problema. Veja os resultados a seguir: 
Tabela 1 - Resultados estatísticos resumidos

\begin{tabular}{|c|c|c|}
\hline \multirow{2}{*}{ Variável dependente: IFDM } & \multicolumn{2}{|c|}{ Regressões lineares } \\
\hline & Reduzida & Completa \\
\hline \multirow[t]{2}{*}{ Intercepto } & $0,146 * * *$ & $0,298 * * *$ \\
\hline & 0,021 & 0,021 \\
\hline \multirow[t]{2}{*}{ PART } & $0,357 * * *$ & $0,177^{* * *}$ \\
\hline & 0,024 & 0,023 \\
\hline \multirow[t]{2}{*}{ IFGF } & $0,341 * * *$ & $0,171 * * *$ \\
\hline & 0,009 & 0,009 \\
\hline \multirow[t]{2}{*}{ NE } & & $0,021 * * *$ \\
\hline & & 0,005 \\
\hline \multirow[t]{2}{*}{$\mathrm{CO}$} & & $0,110 * * *$ \\
\hline & & 0,006 \\
\hline \multirow[t]{2}{*}{ SU } & & $0,141 * * *$ \\
\hline & & 0,005 \\
\hline \multirow[t]{2}{*}{ SD } & & $0,150 * * *$ \\
\hline & & 0,009 \\
\hline $\mathrm{N}$ & 5.199 & 5.199 \\
\hline$R^{2}$ & 0,243 & 0,477 \\
\hline AIC & $-9.251,736$ & $-11.140,49$ \\
\hline $\mathrm{BIC}$ & $-9.232,114$ & $-11.094,71$ \\
\hline Root MSE & 0,098 & 0,081 \\
\hline Estatística F & $987,82^{* * *}$ & $805,70 * * *$ \\
\hline
\end{tabular}

Todos os modelos são significativos, ou seja, as variáveis participação social e gestão fiscal explicam desenvolvimento social. Observa-se que a explicação do modelo $\left(R^{2}\right)$ aumenta consideravelmente da regressão reduzida para a completa. A partir da regressão completa, é possível evidenciar que as variáveis dummies controlam as diferenças de valores de desenvolvimento social entre as regiões 
brasileiras. A partir dos valores de AIC e BIC, bem como o Root MSE, sugere-se que o modelo completo é a melhor opção.

Nota-se que a regressão completa (que considera as variáveis independentes de participação política, gestão fiscal e dummies regionais) explica 47,7\% desses resultados (desenvolvimento social) municipais. Adicionalmente, a partir da análise comparativa das medianas das regiões com a mediana brasileira (Figura 2), constatase o padrão de variações de desenvolvimento social nos municípios entre as regiões brasileiras, sendo que o grupo de municípios das regiões Centro-oeste, Sudeste e Sul apresenta resultados mais prósperos de desenvolvimento social do que os das regiões Norte e Nordeste. Sugere-se que os governantes devem melhorar as iniciativas participativas e de gestão fiscal, assim como aperfeiçoar as instituições orçamentárias considerando a perspectiva regional, com vistas a, principalmente, reduzir a pobreza e, consequentemente, as desiguais sociais brasileiras.

\section{Figura 2 - Comparativo regional de desenvolvimento}

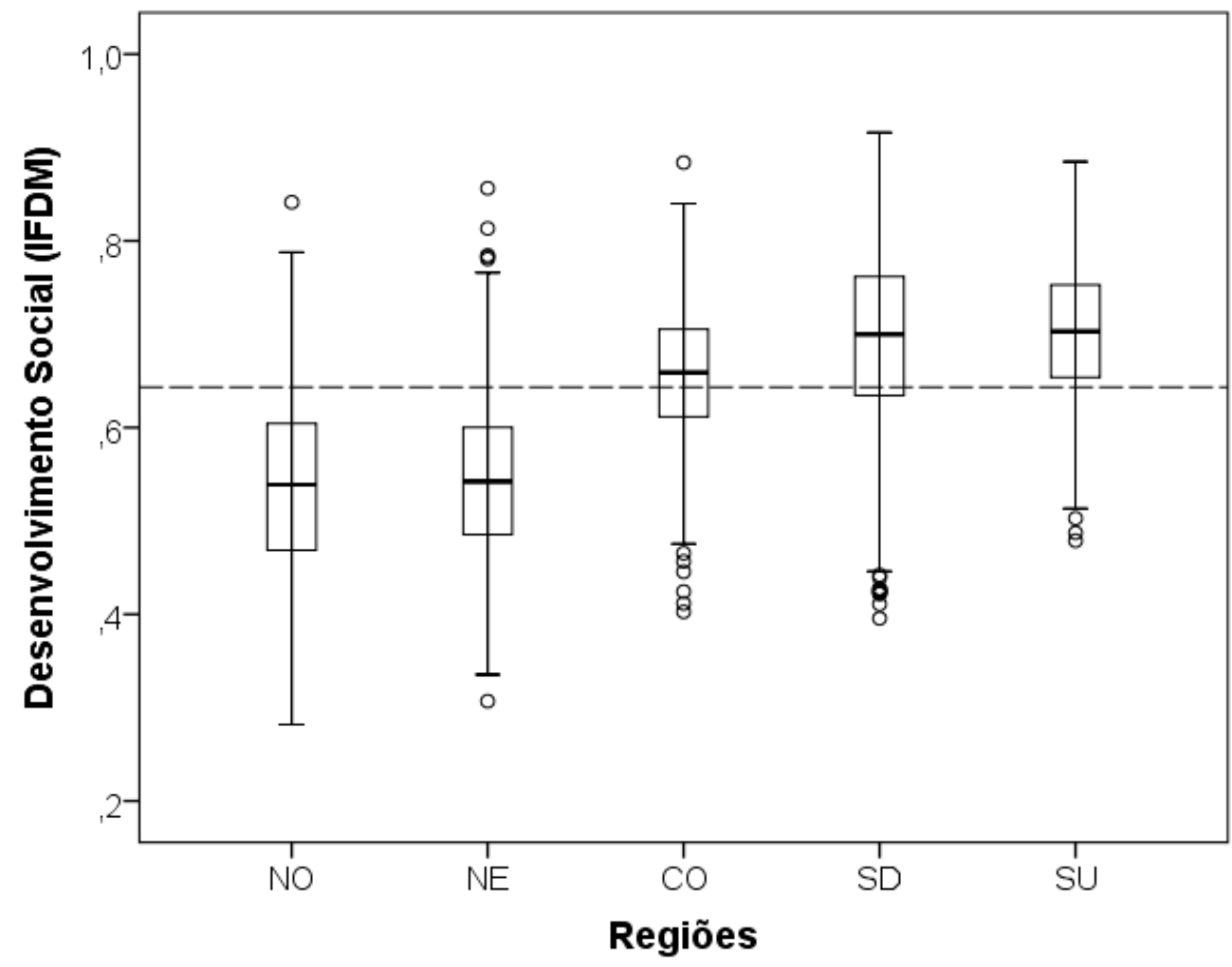

Fonte: Firjan.

Outro fator a ser observado é que o desenvolvimento social local poderia gerar um aumento cíclico na participação (virtuoso), expandindo os seus efeitos na gestão 
fiscal e, consequentemente, no desenvolvimento social. Alguns estudos indicam que a apatia política pode comprometer o processo democrático e que cidadãos como menos níveis de escolaridade e menor renda tendem a participar menos da vida política (BobBIO; BOVERo, 1986; AltMAN; Pérez-LiÑ́́N, 2002).

Embora o modelo deste estudo observe a relação participação política e gestão fiscal sobre o desenvolvimento social local, ele também aponta caminhos para estudos futuros que poderiam comprovar empiricamente, por exemplo, que: desenvolvimento social baseado em distribuição de renda e educação deve ser capaz de gerar participação social e melhorias na gestão fiscal.

Dessa forma, pode-se esperar que, através de evidências empíricas, os municípios com melhores valores de desenvolvimento social estejam relacionados com maior participação política e melhor gestão fiscal. Assim como ficou comprovado que o controle regional contribui significativamente para explicar os resultados de desenvolvimento social local, conforme sugere a literatura revisada nesta pesquisa.

\section{Considerações finais}

A partir dos resultados desta pesquisa, espera-se reduzir a lacuna de conhecimento sobre as relações do desenvolvimento social com a participação política e a gestão fiscal. Em especial, aprender sobre como os municípios brasileiros variam com base nas referidas variáveis, assim como se comportam regionalmente.

Com base nos referidos resultados, pode-se encontrar evidências empíricas validando a existência de relação positiva do desenvolvimento social com a participação política e a gestão fiscal. Após a adição de variável controle relacionada com a questão regional, identificou-se a ocorrência de ganhos significativos de explicação do modelo em estudo.

Dessa forma, verifica-se que as políticas públicas que promovem o desenvolvimento social, em especial as relacionadas com incentivos à participação política e de gestão fiscal, precisam ser estimuladas mais em municípios de algumas regiões do que outras, como forma de estímulo à equidade regional e à justiça social.

Embora ambas as regressões, a reduzida e a completa, sejam significativas estatisticamente, observa-se que a regressão completa é a opção melhor, não só tendo em vista o incremento no poder de explicação entre os modelos, mas também considerando os respectivos valores dos testes AIC, BIC e Root MSE. Isso enfatiza, ainda mais, a relevância das variáveis dummies para controlar as diferenças entre os dados municipais por regiões brasileiras.

Espera-se, assim, ter contribuído para consolidação e expansão da presente temática. Em tempo, ressalta-se a importância de se realizarem novas pesquisas 
com vistas a analisar qualitativamente as conclusões desta pesquisa, bem como buscar novas evidências que podem colaborar ou contrapor os resultados apresentados neste artigo.

Assim sendo, este artigo contribui para a validação do modelo teórico proposto, de maneira a concluir que o engajamento político dos cidadãos, assim como gestão fiscal responsável colaboram com a redução das desigualdades regionais, e, portanto, contribuindo para a explicação do desenvolvimento social.

Diante do exposto, instituições participativas politicamente e responsáveis do ponto de vista fiscal, além de melhorarem a qualidade democrática, apresentam um círculo virtuoso buscando a redução da pobreza, rumo ao desenvolvimento social. Por fim, destaca-se a importância de considerar questões regionais como fundamentais para a diminuição das desigualdades sociais.

\section{Referências bibliográficas}

ABBAD, G.; TORRES, C. V. Regressão múltipla stepwise e hierárquica em Psicologia Organizacional: aplicações, problemas e soluções. Estudos de Pscicologia, v. 7, p. 19-29, 2002.

Abreu, W. M. Orçamento público. Brasília, DF: Enap, 2013.

Albuquerque, C. M.; Medeiros, M.; Feisó, P. H. Gestão de finanças públicas: fundamentos e práticas de planejamento, orçamento e administração financeira com responsabilidade fiscal. 1. Brasília, DF: 2006.

AltMAN, D.; Pérez-LiÑÁN, A. Assessing the quality of democracy: freedom, competitiveness and participation in eighteen Latin American countries. Democratization, v. 9, n. 2, p. 85-100, 2002.

ANSELL, C.; GASH, A. Collaborative governance in theory and practice. Journal of Public Administration Research and Theory, v. 18, n. 4, p. 543-571, 2008.

AVRItZer, L.; NaVARro, Z. A inovação democrática: o orçamento participativo no Brasil. São Paulo: Cortez 2003.

BAIOCCHI, G. et al. Evaluating empowerment: participatory budgeting in Brazilian municipalities. Empowerment in practice: from analysis to implementation. Washington DC: The World Bank, 2006.

BANDEIRA, P. Participação, articulação de atores sociais e desenvolvimento regional. Brasília: Ipea, 1999.

BARCELOS, C. L. K. Quinze anos sem Aaron Wildavsky: recordando lições valiosas. In: Encontro de Administraçao Pública e Governança. Salvador, BA, 2008.

Bоввıо, N. Liberalismo velho e novo. O futuro da democracia: uma defesa das regras do jogo. Volume 3. 1986.

BobBIO, N.; BovERo, M. Sociedade e Estado na filosofia política moderna. Brasiliense, 1986. 
BovaIRD, T. Public governance: balancing stakeholder power in a network society. International Review of Administrative Sciences, v. 71, n. 2, p. 217-228, 2005.

BRASIL. Manual técnico de orçamento, MTO 2014. Brasília, DF: Ministério do Planejamento, Orçamento e Gestão. Secretaria de Orçamento Federal, 2013. http://www.ibge.gov.br/home/. 2014. Acesso em: 14/04/2014.

Bühlmann, M. et al. The democracy barometer: a new instrument to measure the quality of democracy and its potential for comparative research. European Political Science, v. 11, n. 4, p. 519-536, 2012.

CABANNES, Y. Participatory budgeting: a significant contribution to participatory democracy. Environment and Urbanization, v. 16, n. 1, p. 27-46, 2004.

CARA, D. Municípios no pacto federativo - fragilidades sobrepostas. Retratos da Escola, v. 6, n. 10, 2012.

Coelho, V. S. P.; Nobre, M. Participação e deliberação: teoria democrática e experiências institucionais no Brasil contemporâneo. Editora 34, 2004.

CORE, F. G. Reformas orçamentárias no Brasil: uma trajetória de tradição e formalismo na alocação dos recursos públicos. In: CONGRESO INTERNACIONAL DEL CLAD SOBRE LA Reforma del Estado Y DE LA AdMinistración PúBliCA, 9., 2004, Madrid. Anais... Madrid, España: CLAD 2004.

CRUz, C. F. et al. Transparência da gestão pública municipal: um estudo a partir dos portais eletrônicos dos maiores municípios brasileiros. Revista de Administração Pública, v. 46, n. 1, p. 153-76, 2012.

DAHL, R. A democracia e seus críticos. São Paulo: Fontes, 2012.

DAVIS, G. A history of the social development network in The World Bank, 19732003. The World Bank, 2004.

DEPARTAMENT FOR INTERNATIONAL DEVELOPMENT (DFID). Governance, development and democratic politics. DFID, 2006.

DiAmond, L. J.; MoRLINo, L. An overview. Journal of Democracy, v. 15, n. 4, p. 20-31, 2004.

EMERSON, K.; MURCHIE, P. Collaborative governance and climate change. The future of public administration around the world: the Minnowbrook perspective, p. 141, 2011.

EMERSON, K.; NABATCHI, T.; BALOGH, S. An integrative framework for collaborative governance. Journal of Public Administration Research and Theory, v. 22, n. 1, p. 1-29, 2012.

FEDERAÇÃO DE INDÚSTRIAS DO ESTADO DO RIO DE JANEIRO (FIRJAN). http://www.firjan. org.br/. 2014. Acesso em: 14/04/2014.

FREEMAN, R. E. Strategic management: a stakeholder approach. Massachusetts: Pitman, 1984.

. The politics of stakeholder theory: some future directions. Business Ethics Quarterly, p. 409-421, mar-abr 1994.

Frils-HANSEN, E.; Cold-RAVNKILDE, S. M. Social accountability mechanisms and 
access to public service delivery in rural Africa. DIIS Reports, Danish Institute for International Studies. Copenhagen, p. 96. 2013.

Frils-Hansen, E.; KYED, H. M. Participation, decentralization and human rights: a review of approaches for strengthening voice and accountability in local governance. Washington, DC: WB, 2009.

GARCIA, R. C. A reorganização do processo de planejamento do Governo Federal: o PPA 2000-2003. Texto para Discussão IPEA n. 726. Brasília: Ipea, maio 2000.

GAVENTA, J.; MCGEE, R. The impact of transparency and accountability initiatives. Development Policy Review, v. 31, n. s1, p. s3-s28, 2013.

GRUBER, J. Finanças públicas e política pública. 2. ed. Rio de janeiro: LTC, 2009.

HABERMAS, J. Teoria do agir comunicativo. São Paulo: Editora WMF Martins Fortes, 2012.

HYDE, A. C. Government budgeting: theory, process, and politics. Wadsworth Publishing Company, 2002.

KASYMOVA, J. T.; SCHACHTER, H. L. Bringing participatory tools to a different level. Public Performance \& Management Review, v. 37, n. 3, p. 441-464, 2014.

Khagram, S.; Fung, A.; De Renzio, P. Open budgets: the political economy of transparency, participation, and accountability. Washington, DC: Brookings Institution Press, 2013.

LAWRENCE, R. Z. Is it really the economy, stupid? In: NYE, J. S.; ZeLIKOW, P.; KING, D. C. Why people don't trust government. Harvard University Press, 1997. p. 111-132.

LIJPHART, A. Modelos de democracia: desempenho e padrões de governo em 36 países. trad. de Roberto Franco. Rio de Janeiro: Civilização Brasileira, 2003.

LING, C.; ROBERTS, D. K. Evidence of development impact from institutional change: a review of the evidence on open budgeting. Washington, DC: WB, 2014. p. 32.

Marquetti, A. et al. Democracia participativa e redistribuição: análise de experiências de orçamento participativo. Xamã, 2008.

MITCHELL, R. K.; AGLE, B. R.; WoOD, D. J. Toward a theory of stakeholder identification and salience: defining the principle of who and what really counts. Academy of Management Review, v. 22, n. 4, p. 853-886, 1997.

NAVARRO, Z. Decentralization, participation and social control of public resources: participatory budgeting in Porto Alegre (Brazil). IADB (online), 2005. Disponível em: http://www.iadb.org/int/jpn/english/support_files/9brazil.pdf. Acesso em: 05/06/2006.

NORTH, D. Institutions Journal of Economic Perspectives. Cambridge: American Economic Association. 1: 97-112 p. 1991.

NORTH, D. C. Institutions, institutional change and economic performance. Cambridge university press, 1990.

NUNES, G. S. D. F. et al. Análise dos instrumentos de transparência contidos na Lei de Responsabilidade Fiscal nos Municípios da Região Sul. Revista Ambiente Contábil, Universidade Federal do Rio Grande do Norte, v. 5, n. 2, p. 128-150, 2013. ISSN 2176-9036 
NYE, J. S.; ZelikoW, P.; KING, D. C. Why people don't trust government. Harvard University Press, 1997.

PADGETT, J. Bounded rationality in budgetary research. American Political Science Review, v. 74, p. 354 - 372, 1980.

Pateman, C. Participação e teoria democrática. Paz e Terra, 1992.

PRovAN, K. G.; KENIS, P. Modes of network governance: structure, management, and effectiveness. Journal of Public Administration Research and Theory, v. 18, n. 2, p. 229-252, 2008.

Provan, K. G.; MilWARD, H. B. A preliminary theory of interorganizational network effectiveness: a comparative study of four community mental health systems. Administrative Science Quarterly, p. 1-33, 1995.

Rodriguez, J. R.; Vitale, D.; Melo, R. S. Participação e deliberação na teoria democrática: uma introdução. Participação e deliberação: teoria democrática e experiências institucionais no Brasil contemporâneo, p. 21, 2004.

SANTOS, W. G. D. et al. Cidadania e participação. 2005.

SCHNEIDER, A.; BAQUERO, M. Get what you want, give what you can: embedded public finance in Porto Alegre. Institute of Development Studies, University of Sussex, 2006.

SCHROEDER, U. C. Measuring security sector governance: a guide to relevant indicators. Genebra: Geneva Centre for the Democratic Control of Armed Forces (DCAF), 2010. p.66.

SHAH, A. Participatory budgeting. Washington, DC: WB, 2007.

SILVA, L. D. A influência do desempenho tributário e gestão fiscal no índice Firjan de desenvolvimento (IFDM) dos municípios de Minas Gerais. Revista de C. Humanas, p. 199-219, 2013.

Slaibe Postali, F. A.; NISHIJIMA, M. Distribuição das rendas do petróleo e indicadores de desenvolvimento municipal no Brasil nos anos 2000'. Estudos Econômicos, v. 41, n. 2, p. 463-485, 2011.

StokeR, G. Governance as theory: five propositions. International Social Science Journal, v. 50, n. 155, p. 17-28, 1998.

SWAIN, J. W.; HARTLEY JR, C. J. Incrementalism: old but good? In: BARTLE, J. R. (Ed.). Evolving theories of public budgeting. Volume 6. Amsterdam: Elsevier Science, 2001. p. 11-27.

THE ECONOMIST. Democracy Index, 2014. Disponível em: http://www.eiu.com/public/ topical_report.aspx?campaignid=Democracylndex12. Acesso em: 11/10/2014.

TOUCHTON, M.; WAMPLER, B. Improving social well-being through new democratic institutions. Comparative Political Studies, v. 47, n. 10, p. 1442-1469, 2014.

WAMPLER, B. Expanding accountability through participatory institutions: mayors, citizens, and budgeting in three Brazilian municipalities. Latin American Politics and Society, v. 46, n. 2, p. 73-99, 2004. 
Participation, transparency and accountability: innovations in South Korea, Brazil, and the Philippines. GIFT, p. 61, 2012.

WildaVsky, A.; DeMPSTER, M.; DAVIS, O. A theory of the budgetary process. American Political Science Review, v. LX, n. 8, p. 529 - 547, Setembro 1966.

WORLD BANK (WB). http://info.worldbank.org/governance/wgi/index.aspx\#home. 2014. Acesso em: 11/10/2014.

YILMAZ, S.; BeRIS, Y.; SERRANO-BERTHET, R. Linking local government discretion and accountability in decentralisation. Development Policy Review, v. 28, n. 3, p. 259293, 2010.

Welles Matias de Abreu

Contato: wellesmatias@hotmail.com

Ricardo Corrêa Gomes

Contato: rgomes@unb.br

Daniel Cerqueira Ribeiro

Contato: daniel.dcr09@gmail.com

José Matias-Pereira

Contato:matias@unb.br

Fábio Jacinto Barreto de Souza

Contato: fabiojjsouza@gmail.com 